\title{
A POSITIVE GRASSMANNIAN ANALOGUE OF THE PERMUTOHEDRON
}

\author{
LAUREN K. WILLIAMS
}

\begin{abstract}
The classical permutohedron $\operatorname{Perm}_{n}$ is the convex hull of the points $(w(1), \ldots, w(n)) \in \mathbb{R}^{n}$ where $w$ ranges over all permutations in the symmetric group $S_{n}$. This polytope has many beautiful properties for example it provides a way to visualize the weak Bruhat order: if we orient the permutohedron so that the longest permutation $w_{0}$ is at the "top" and the identity $e$ is at the "bottom," then the one-skeleton of $\operatorname{Perm}_{n}$ is the Hasse diagram of the weak Bruhat order. Equivalently, the paths from $e$ to $w_{0}$ along the edges of $\mathrm{Perm}_{n}$ are in bijection with the reduced decompositions of $w_{0}$. Moreover, the two-dimensional faces of the permutohedron correspond to braid and commuting moves, which by the Tits Lemma, connect any two reduced expressions of $w_{0}$.

In this note we introduce some polytopes $\mathrm{Br}_{k, n}$ (which we call bridge polytopes) which provide a positive Grassmannian analogue of the permutohedron. In this setting, BCFW bridge decompositions of reduced plabic graphs play the role of reduced decompositions. We define $\operatorname{Br}_{k, n}$ and explain how paths along its edges encode BCFW bridge decompositions of the longest element $\pi_{k, n}$ in the circular Bruhat order. We also show that two-dimensional faces of $\mathrm{Br}_{k, n}$ correspond to certain local moves for plabic graphs, which by a result of Postnikov [Pos06], connect any two reduced plabic graphs associated to $\pi_{k, n}$. All of these results can be generalized to the positive parts of Schubert cells. A useful tool in our proofs is the fact that our polytopes are isomorphic to certain Bruhat interval polytopes. Conversely, our results on bridge polytopes allow us to deduce some corollaries about the structure of Bruhat interval polytopes.
\end{abstract}

\section{CONTENTS}

1. Introduction

2. Background

3. Bridge polytopes and their one-skeleta

4. Bruhat interval polytopes and the proof of Theorem 3.2

5. The two-dimensional faces of bridge polytopes

References

\section{INTRODUCTION}

The totally nonnegative part $\left(G r_{k, n}\right)_{\geq 0}$ of the real Grassmannian $G r_{k, n}$ is the locus where all Plücker coordinates are non-negative Lus94, Rie99, Pos06]. Postnikov initiated the combinatorial study of $\left(G r_{k, n}\right)_{\geq 0}$ : he showed that if one stratifies the space based on which Plücker coordinates are positive and which are zero, one gets a cell decomposition. The cells are in bijection with several families of combinatorial objects, including decorated permutations, and equivalence classes of reduced plabic graphs. Reduced plabic graphs are a certain family of planar bicolored graphs embedded in a disk. These graphs are very interesting: they can be used to parameterize cells in $\left(G r_{k, n}\right)_{\geq 0}$ Pos06] and to understand the cluster algebra structure on the Grassmannian [Sco06; they also arise as soliton graphs associated to the KP hierarchy [KW11. Most recently reduced plabic graphs and the totally non-negative Grassmannian $\left(G r_{k, n}\right)_{\geq 0}$ have been studied in connection with scattering amplitudes in $N=4$ super Yang-Mills $\mathrm{AHBC}^{+} 12$. Motivated by physical considerations, the authors gave a systematic way for building up a reduced plabic graph for a given cell of $\left(G r_{k, n}\right)_{\geq 0}$, which they called the BCFW-bridge construction.

In many ways, reduced plabic graphs behave like reduced decompositions of the symmetric group: for example, just as any two reduced decompositions of the same permutation can be related by braid and commuting moves, any two reduced plabic graphs associated to the same decorated permutation can be

Date: January 6, 2015.

The author was partially supported by an NSF CAREER award DMS-1049513. 
related via certain local moves [Pos06]. The goal of this note is to highlight another way in which reduced plabic graphs behave like reduced decompositions. We will introduce a polytope called the bridge polytope, which is a positive Grassmannian analogue of the permutohedron in the following sense: just as the oneskeleton of the permutohedron encodes reduced decompositions of permutations, the one-skeleton of the bridge polytope encodes BCFW-bridge decompositions of reduced plabic graphs. Moreover, just as the two-dimensional faces of permutohedra encode braid and commuting moves for reduced decompositions, the two-dimensional faces of bridge polytopes encode local moves for reduced plabic graphs.

Acknowledgments: The author is grateful to Nima Arkani-Hamed, Jacob Bourjaily, and Yan Zhang for conversations concerning the BCFW bridge construction.

\section{BACKGROUND}

2.1. Reduced decompositions, Bruhat order, and the permutohedron. Recall that the symmetric group $S_{n}$ is the group of all permutations on $n$ letters. If we let $s_{i}$ denote the simple transposition exchanging $i$ and $i+1$, then $S_{n}$ is a Coxeter group generated by the $s_{i}$ (for $1 \leq i<n$ ), subject to the relations $s_{i}^{2}=1$, as well as the commuting relations $s_{i} s_{j}=s_{j} s_{i}$ for $|i-j| \geq 2$ and braid relations $s_{i} s_{i+1} s_{i}=s_{i+1} s_{i} s_{i+1}$.

Given $w \in S_{n}$, there are many ways to write $w$ as a product of simple transpositions. If $w=s_{i_{1}} \ldots s_{i_{\ell}}$ is a minimal-length such expression, we call $s_{i_{1}} \ldots s_{i_{\ell}}$ a reduced expression of $w$, and $\ell$ the length $\ell(w)$ of $w$. The well-known Tits Lemma asserts that any two reduced expressions can be related by a sequence of braid and commuting moves if and only if they are reduced expressions for the same permutation.

There are two important partial orders on $S_{n}$, both of which are graded, with rank function given by the length of the permutation. The strong Bruhat order is the transitive closure of the cover relation $u \prec v$, where $u \prec v$ means that $(i j) u=v$ for $i<j$ and $\ell(v)=\ell(u)+1$. Here $(i j)$ is the transposition (not necessarily simple) exchanging $i$ and $j$. We use the symbol $\preceq$ to indicate the strong Bruhat order. The strong Bruhat order has many nice combinatorial properties: it is thin and shellable, and hence is a regular CW poset Ede81, Pro82, BW82. Moreover, this partial order is closely connected to the geometry of the complete flag variety $\mathrm{FI}_{n}$ : it encodes when one Schubert cell is contained in the closure of another. Somewhat less well-known is its connection to total positivity. The totally non-negative part $U_{>0}^{+}$of the unipotent part of $G L_{n}$ has a decomposition into cells, indexed by permutations, and the strong Bruhat order describes when one cell is contained in the closure of another [Lus94.

The other partial order associated to $S_{n}$ is the (left) weak Bruhat order. This is the transitive closure of the cover relation $u \lessdot v$, where $u \lessdot v$ means that $s_{i} u=v$ and $\ell(v)=\ell(u)+1$. We use the symbol $\leq$ to indicate the weak Bruhat order. The weak Bruhat order is related to reduced decompositions in the following way: given any $w \in S_{n}$, the maximal chains from the identity $e$ to $w$ in the weak Bruhat order are in bijection with reduced decompositions of $w$. Moreover, the weak order has the advantage of being conveniently visualized in terms of a polytope called the permutohedron, as we now describe.

Definition 2.1. The permutohedron $\operatorname{Perm}_{n}$ in $\mathbb{R}^{n}$ is the convex hull of the points $\left\{(z(1), \ldots, z(n)) \mid z \in S_{n}\right\}$.

See Figure 1 for the permutohedron Perm 4 . We remark that the permutohedron is also connected to the geometry of the complete flag variety $\mathrm{FI}_{n}$ : it is the image of $\mathrm{FI}_{n}$ under the moment map.

Given a permutation $z \in S_{n}$, we will often refer to it using the notation $(z(1), \ldots, z(n))$, or $\left(z_{1}, \ldots, z_{n}\right)$, where $z_{i}$ denotes $z(i)$.

The following proposition is well-known, see e.g. $\mathrm{BLVS}^{+}$99.

Proposition 2.2. Let $u$ and $v$ be permutations in $S_{n}$ with $\ell(u) \leq \ell(v)$. There is an edge between vertices $u$ and $v$ in the permutohedron $\operatorname{Perm}_{n}$ if and only if $v=s_{i} u$ for some $i$ and $\ell(v)=\ell(u) \pm 1$, in which case we label the corresponding edge of the permutohedron by $s_{i}$. Moreover, the two-dimensional faces of Perm $_{n}$ are either squares (with edges labeled in an alternating fashion by $s_{i}$ and $s_{j}$, where $|i-j| \geq 2$ ) or hexagons (with edges labeled in an alternating fashion by $s_{i}$ and $s_{i+1}$ for some $i$ ).

See Figure 1 for the example of Perm 4 . Proposition 2.2 implies that edges of the permutohedron correspond to cover relations in the weak Bruhat order, and the two-dimensional faces correspond to braid and commuting relations for reduced words.

Let $w_{0}$ be the longest permutation in $S_{n}$. This permutation is unique, and can be defined by $w_{0}(i)=$ $n+1-i$. Proposition 2.2 can be equivalently restated as follows. 


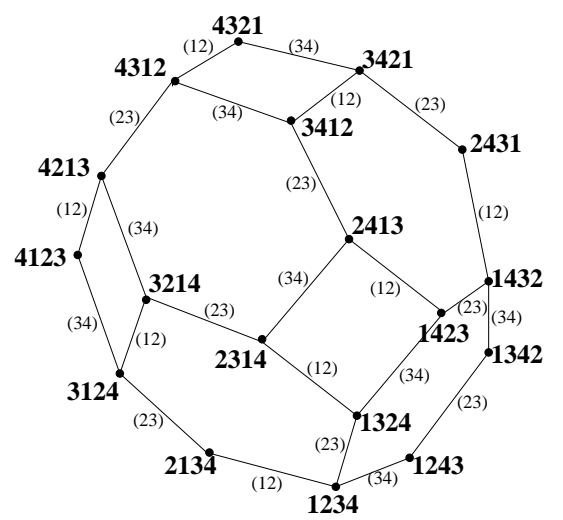

Figure 1. The permutohedron Perm 4 . Edges are labeled by the values swapped when we go between the permutations labeling the two vertices.

Proposition 2.3. The shortest paths from e to $w_{0}$ along the one-skeleton of the permutohedron Perm $_{n}$ are in bijection with the reduced decompositions of $w_{0}$.

For example, we can see from Figure 1 that there is a path

$$
e \lessdot(2,1,3,4) \lessdot(3,1,2,4) \lessdot(4,1,2,3) \lessdot(4,2,1,3) \lessdot(4,3,1,2) \lessdot(4,3,2,1)=w_{0}
$$

in the permutohedron $\mathrm{Perm}_{4}$. Reading off the edge labels of this path gives rise to the reduced decomposition $s_{1} s_{2} s_{1} s_{3} s_{2} s_{1}$ of $w_{0}$.

2.2. The positive Grassmannian, permutations, circular Bruhat order, and plabic graphs. In this section we introduced the positive Grassmannian, and some combinatorial objects associated to it, including permutations and plabic graphs. As we will see, reduced plabic graphs are in many ways analogous to reduced decompositions of permutations.

The real Grassmannian $G r_{k, n}$ is the space of all $k$-dimensional subspaces of $\mathbb{R}^{n}$. An element of $G r_{k, n}$ can be viewed as a full-rank $k \times n$ matrix modulo left multiplication by nonsingular $k \times k$ matrices. In other words, two $k \times n$ matrices represent the same point in $G r_{k, n}$ if and only if they can be obtained from each other by row operations. Let $\left(\begin{array}{c}{[n]} \\ k\end{array}\right)$ be the set of all $k$-element subsets of $[n]:=\{1, \ldots, n\}$. For $I \in\left(\begin{array}{c}{[n]} \\ k\end{array}\right)$, let $\Delta_{I}(A)$ be the Plücker coordinate, that is, the maximal minor of the $k \times n$ matrix $A$ located in the column set $I$. The map $A \mapsto\left(\Delta_{I}(A)\right)$, where $I$ ranges over $\left(\begin{array}{c}{[n]} \\ k\end{array}\right)$, induces the Plücker embedding $\left.G r_{k, n} \hookrightarrow \mathbb{R} \mathbb{P}^{n} \begin{array}{c}n \\ k\end{array}\right)-1$. The totally non-negative part of the Grassmannian (sometimes called the positive Grassmannian) $\left(G_{k, n}\right)_{\geq 0}$ is the subset of $G r_{k, n}$ such that all Plücker coordinates are non-negative.

If we partition $\left(G r_{k, n}\right)_{\geq 0}$ into strata based on which Plücker coordinates are positive and which are zero, we obtain a decomposition into positroid cells Pos06. Postnikov showed that the cells are in bijection with, and naturally labeled by, several families of combinatorial objects, including Grassmann necklaces, decorated permutations and equivalence classes of reduced plabic graphs. He also introduced the circular Bruhat order on decorated permutations, which describes when one cell is contained in another. Like the strong Bruhat order, the circular Bruhat order is graded, thin, shellable, and hence is a regular CW poset Wil07.

Definition 2.4. A Grassmann necklace is a sequence $\mathcal{I}=\left(I_{1}, \ldots, I_{n}\right)$ of subsets $I_{r} \subset[n]$ such that, for $i \in[n]$, if $i \in I_{i}$ then $I_{i+1}=\left(I_{i} \backslash\{i\}\right) \cup\{j\}$, for some $j \in[n]$; and if $i \notin I_{r}$ then $I_{i+1}=I_{i}$. (Here indices $i$ are taken modulo n.) In particular, we have $\left|I_{1}\right|=\cdots=\left|I_{n}\right|$, which is equal to some $k \in[n]$. We then say that $\mathcal{I}$ is a Grassmann necklace of type $(k, n)$.

To construct the Grassmann necklace associated to a positroid cell, one uses the following construction.

Lemma 2.5. Define the shifted linear order $<_{i}$ by $i<_{i} i+1<_{i} \cdots<_{i} n<_{i} 1<_{i} \cdots<_{i} i-1$. Given $A \in G r_{k, n}$, let $\mathcal{I}(A)=\left(I_{1}, \ldots, I_{n}\right)$ be the sequence of subsets in $[n]$ such that, for $i \in[n], I_{i}$ is the lexicographically minimal subset of $\left(\begin{array}{c}{[n]} \\ k\end{array}\right)$ with respect to the shifted linear order such that $\Delta_{I_{i}}(A) \neq 0$. Then $\mathcal{I}(A)$ is a Grassmann necklace of type $(k, n)$. 
Definition 2.6. A decorated permutation $\pi$ on $n$ letters is a permutation on $n$ letters in which fixed points have one of two colors, "clockwise" and "counterclockwise". A position $i$ of $\pi$ is called a weak excedance if $\pi(i)>i$ or $\pi(i)=i$ and $i$ is a counterclockwise fixed point.

Definition 2.7. Given a Grassmann necklace $\mathcal{I}$, define a decorated permutation $\pi=\pi(\mathcal{I})$ by requiring that

(1) if $I_{i+1}=\left(I_{i} \backslash\{i\}\right) \cup\{j\}$, for $j \neq i$, then $\pi(j)=i$.

(2) if $I_{i+1}=I_{i}$ and $i \in I_{i}$ then $\pi(i)=i$ is a counterclockwise fixed point.

(3) if $I_{i+1}=I_{i}$ and $i \notin I_{i}$ then $\pi(i)=i$ is a clockwise fixed point.

As before, indices are taken modulo $n$.

Using the above constructions, one may show the following.

Theorem 2.8. Pos06 The cells of $\left(G r_{k, n}\right)_{\geq 0}$ are in bijection with Grassmann necklaces of type $(k, n)$ and with decorated permutations on $n$ letters with exactly $k$ weak excedances.

The totally non-negative Grassmannian $\left(G r_{k, n}\right)_{\geq 0}$ has a unique top-dimensional cell, consisting of elements where all Plücker coordinates are strictly positive. This subset is called the totally positive Grassmannian $\left(G r_{k, n}\right)_{>0}$, and it is labeled by the decorated permutation $\pi_{k, n}:=(n-k+1, n-k+2, \ldots, n, 1,2, \ldots, n-k)$. More generally, there is a nice class of positroid cells called the TP or totally positive Schubert cells.

Definition 2.9. A TP Schubert cell is the unique positroid cell of greatest dimension which lies in the intersection of a usual Schubert cell with $\left(G r_{k, n}\right)_{\geq 0}$. The TP Schubert cells in $\left(G r_{k, n}\right)_{\geq 0}$ are in bijection with $k$-element subsets $J$ of $[n]=\{1,2, \ldots, n\}$. To calculate the decorated permutation associated to the TP Schubert cell indexed by $J$, write $J=\left\{j_{1}<\cdots<j_{k}\right\}$, and the complement of $J$ as $J^{c}=\left\{h_{1}<\cdots<h_{n-k}\right\}$. Then the corresponding decorated permutation $\pi=\pi(J)$ is defined by $\pi\left(h_{i}\right)=i$ for all $1 \leq i \leq n-k$, and $\pi\left(j_{i}\right)=n-k+i$ for all $1 \leq i \leq k$. (Any fixed points that arise are considered to be weak excedances if and only if they are in positions labeled by $J$.

A Grassmannian permutation is a permutation $\pi=(\pi(1), \ldots, \pi(n))$ which has at most one descent, i.e. at most one position $i$ such that $\pi(i)>\pi(i+1)$. Note that the permutations of the form $\pi(J)$ defined above are precisely the inverses of the Grassmannian permutations, since $\pi(J)^{-1}=\left(h_{1}, h_{2}, \ldots, h_{n-k}, j_{1}, j_{2}, \ldots, j_{k}\right)$.

In the case that $J=\{1,2, \ldots, k\}$, the corresponding TP Schubert cell is $\left(G r_{k, n}\right)_{>0}$ and $\pi(J)=\pi_{k, n}$.

To describe the partial order on positroid cells, it is convenient to represent each decorated permutation by a related affine permutation, as was done in KLS13. Then, the circular Bruhat order for $\left(G r_{k, n}\right)_{\geq 0}$ can be viewed as the restriction of the affine Bruhat order to the corresponding affine permutations, together with a new bottom element 0 added to the poset.

Definition 2.10. Given a decorated permutation $\pi$ on $n$ letters, we construct its affinization $\tilde{\pi}$ as follows. If $\pi(i)<i$, set $\tilde{\pi}(i):=\pi(i)+n$. If $\pi(i)=i$ and $i$ is a clockwise fixed point, set $\tilde{\pi}(i):=i$. If $\pi(i)=i$ and $i$ is a counterclockwise fixed point, set $\tilde{\pi}(i):=i+n$. Finally, if $\pi(i)>i$, set $\tilde{\pi}(i):=\pi(i)$.

Clearly the affine permutation constructed above is a map from $\{1,2, \ldots, n\}$ to $\{1,2, \ldots, 2 n\}$ such that $i \leq \tilde{\pi}(i) \leq i+n$. And modulo $n$, it agreeds with the underlying permutation $\pi$.

We now discuss plabic graphs, which e.g. are useful for constructing parameterizations of positroid cells Pos06, and for understanding the cluster structure on the Grassmannian [Sco06].

Definition 2.11. A planar bicolored graph (or plabic graph) is an undirected graph $G$ drawn inside a disk (and considered modulo homotopy) plus $n$ boundary vertices on the boundary of the disk, labeled $1, \ldots, n$ in clockwise order. The remaining internal vertices are strictly inside the disk and are colored in black and white. We require that each boundary vertex is incident to a single edge.

We will always assume that a plabic graph is leafless, i.e. that it has no non-boundary leaves, and that it has no isolated components. The following map gives a connection between plabic graphs and decorated permutations.

Definition 2.12. Given a plabic graph $G$, the trip $T_{i}$ is the directed path which starts at the boundary vertex $i$, and follows the "rules of the road": it turns right at a black vertex and left at a white vertex. Note that $T_{i}$ will also end at a boundary vertex. The decorated trip permutation $\pi_{G}$ is the permutation such that $\pi_{G}(i)=j$ whenever $T_{i}$ ends at $j$. Moreover, if there is a white (respectively, black) boundary leaf at boundary vertex $i$, then $\pi_{G}(i)=i$ is a counterclockwise (respectively, clockwise) fixed point. 


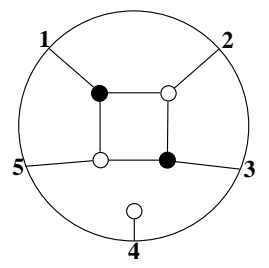

Figure 2. A plabic graph

We now define some local transformations of plabic graphs, which are analogous in some ways to braid and commuting moves for reduced expressions in the symmetric group.

(M1) SQUARE MOVE. If a plabic graph has a square formed by four trivalent vertices whose colors alternate, then we can switch the colors of these four vertices.

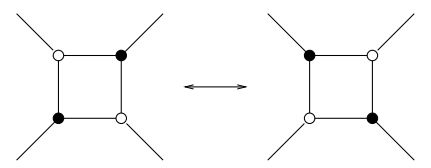

Figure 3. Square move

(M2) UNICOLORED EDGE CONTRACTION/UNCONTRACTION. If a plabic graph contains an edge with two vertices of the same color, then we can contract this edge into a single vertex with the same color. We can also uncontract a vertex into an edge with vertices of the same color.

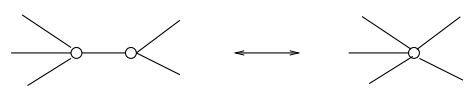

FiguRE 4. Unicolored edge contraction

(M3) MIDDLE VERTEX INSERTION/REMOVAL. If a plabic graph contains a vertex of degree 2, then we can remove this vertex and glue the incident edges together; on the other hand, we can always insert a vertex (of any color) in the middle of any edge.

FiguRE 5. Middle vertex insertion/ removal

(R1) PARALLEL EDGE REDUCTION. If a network contains two trivalent vertices of different colors connected by a pair of parallel edges, then we can remove these vertices and edges, and glue the remaining pair of edges together.

Definition 2.13. Pos06 Two plabic graphs are called move-equivalent if they can be obtained from each other by moves (M1)-(M3). The move-equivalence class of a given plabic graph $G$ is the set of all plabic graphs which are move-equivalent to $G$. A leafless plabic graph without isolated components is called reduced if there is no graph in its move-equivalence class to which we can apply (R1).

The following result is analogous to the Tits Lemma for reduced decompositions of permutations.

Theorem 2.14. PPos06, Theorem 13.4] Two reduced plabic graphs are move-equivalent if and only if they have the same decorated trip permutation.

A priori, it is not so easy to detect whether a given plabic graph is reduced. One characterization was given in Pos06, Theorem 13.2]. Another very simple characterization was given in [KW11].

Definition 2.15. Given a plabic graph $G$ with $n$ boundary vertices, start at each boundary vertex $i$ and label every edge along trip $T_{i}$ with $i$. After doing this for each boundary vertex, each edge will be labeled by up to two numbers (between 1 and $n$ ). If an edge is labeled by two numbers $i<j$, write $[i, j]$ on that edge.

We say that a plabic graph has the resonance property, if after labeling edges as above, the set $E$ of edges incident to a given vertex has the following property: 


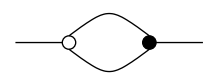

Figure 6. Parallel edge reduction

- there exist numbers $i_{1}<i_{2}<\cdots<i_{m}$ such that when we read the labels of $E$, we see the labels $\left[i_{1}, i_{2}\right],\left[i_{2}, i_{3}\right], \ldots,\left[i_{m-1}, i_{m}\right],\left[i_{1}, i_{m}\right]$ appear in clockwise order.

This property and the following characterization of reduced plabic graphs were given in [KW11.

Theorem 2.16. [KW11, Theorem 10.5] A plabic graph is reduced if and only if it has the resonance property.

2.3. BCFW bridge decompositions. Given a reduced plabic graph, it is easy to construct its corresponding trip permutation, as explained in Definition 2.12. But how can we go backwards, i.e. given the permutation, how can we construct a corresponding reduced plabic graph? One procedure to do this was given in Pos06, Section 20]. Another elegant solution - the BCFW-bridge construction - was given in $\mathrm{AHBC}^{+} 12$, Section 3.2].

To explain the BCFW-bridge construction, we use the representation of each decorated permutation $\pi$ as an affine permutation $\tilde{\pi}$, see Definition 2.10. We say that position $i$ of $\tilde{\pi}$ is a fixed point if $\tilde{\pi}(i)=i$ or $\tilde{\pi}(i)=i+n$. And we say that $\tilde{\pi}$ is a decoration of the identity if it has a fixed point in each position.

Definition 2.17 (The BCFW-bridge construction). Given a decorated permutation $\pi$ on $n$ letters, which is not simply a decoration of the identity, we start by choosing a pair of adjacent positions $i<j$ such that $\tilde{\pi}(i)<\tilde{\pi}(j)$, and $i$ and $j$ are not fixed points. Here adjacent means that either $j=i+1$, or $i<j$ and every position $h$ such that $i<h<j$ is a fixed point. We record the transposition $\tau=(i j)$ and swap the entries in positions $i$ and $j$ in $\tilde{\pi}$. Any entries in the resulting permutation which are fixed points are designated as frozen, and henceforth ignored. We continue this process on the resulting affine permutation, until the end result is a decoration of the identity. Finally we use the sequence of transpositions $\tau$ as a recipe for adding "bridges," thereby constructing a plabic graph.

See Table 1 and Figure 7 for an example of a bridge decomposition of the permutation $\pi=(4,6,5,1,2,3)$ (with corresponding affine permutation $\tilde{\pi}=(4,6,5,7,8,9)$ ). Here a bridge is the subgraph shown at the left of Figure 7 and a bridge decomposition is a graph built by attaching successive bridges. The sequence of transpositions $\tau$ gives a recipe for where to place successive bridges.

\begin{tabular}{|c|llllll|}
\hline & 1 & 2 & 3 & 4 & 5 & 6 \\
$\tau$ & $\downarrow$ & $\downarrow$ & $\downarrow$ & $\downarrow$ & $\downarrow$ & $\downarrow$ \\
\hline$(34)$ & 4 & 6 & 5 & 7 & 8 & 9 \\
$(23)$ & 4 & 6 & 7 & 5 & 8 & 9 \\
$(12)$ & 4 & 7 & 6 & 5 & 8 & 9 \\
$(56)$ & 7 & 4 & 6 & 5 & 8 & 9 \\
$(45)$ & 7 & 4 & 6 & 5 & 9 & 8 \\
$(34)$ & 7 & 4 & 6 & 9 & 5 & 8 \\
$(46)$ & 7 & 4 & 9 & 6 & 5 & 8 \\
\cline { 2 - 6 }$(24)$ & 7 & 4 & 9 & 8 & 5 & 6 \\
\hline & 7 & 8 & 9 & 4 & 5 & 6 \\
\hline
\end{tabular}

TABLE 1. A BCFW-bridge decomposition of $\tilde{\pi}=(4,6,5,7,8,9)$. The frozen entries are boxed.

Proposition 2.18. $\mathrm{AHBC}^{+} 12$, Section 3.2] Given a decorated permutation $\pi$, the BCFW-bridge construction constructs a reduced plabic graph whose trip permutation is $\pi$. 

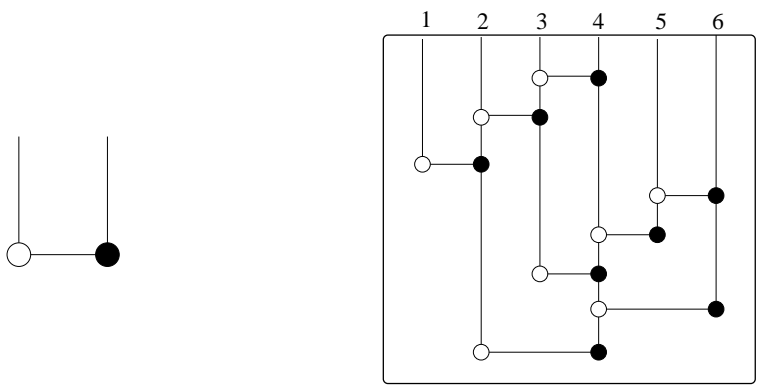

Figure 7. A single bridge, and the plabic graph $G$ associated to the bridge decomposition from Table 1 Note that the trip permutation of $G$ equals the product (24)(46)(34)(45)(56)(12)(23)(34) of transpositions $\tau$ in the bridge decomposition, which equals the corresponding decorated permutation $\pi=(4,6,5,1,2,3)$.

\section{BRIDGE POLYTOPES AND THEIR ONE-SKELETA}

The goal of this section is to introduce some polytopes that we will call bridge polytopes, because their oneskeleta encode BCFW-bridge decompositions of reduced plabic graphs. This statement is analogous to the fact that the one-skeleton of the permutohedron encodes reduced decompositions of $w_{0}$. More specifically, for each $k$-element subset $J$ of $[n]$, we will introduce a bridge polytope $\operatorname{Br}_{J}$ which encodes bridge decompositions of reduced plabic graphs for the permutation $\pi(J)$ labeling the corresponding TP Schubert cell. In the case that $J=\{1,2, \ldots, k\}$, we will also denote $\mathrm{Br}_{J}$ by $\mathrm{Br}_{k, n}$ - this will be the polytope encoding bridge decompositions of $\pi_{k, n}$, the decorated permutation labeling the totally positive Grassmannian.
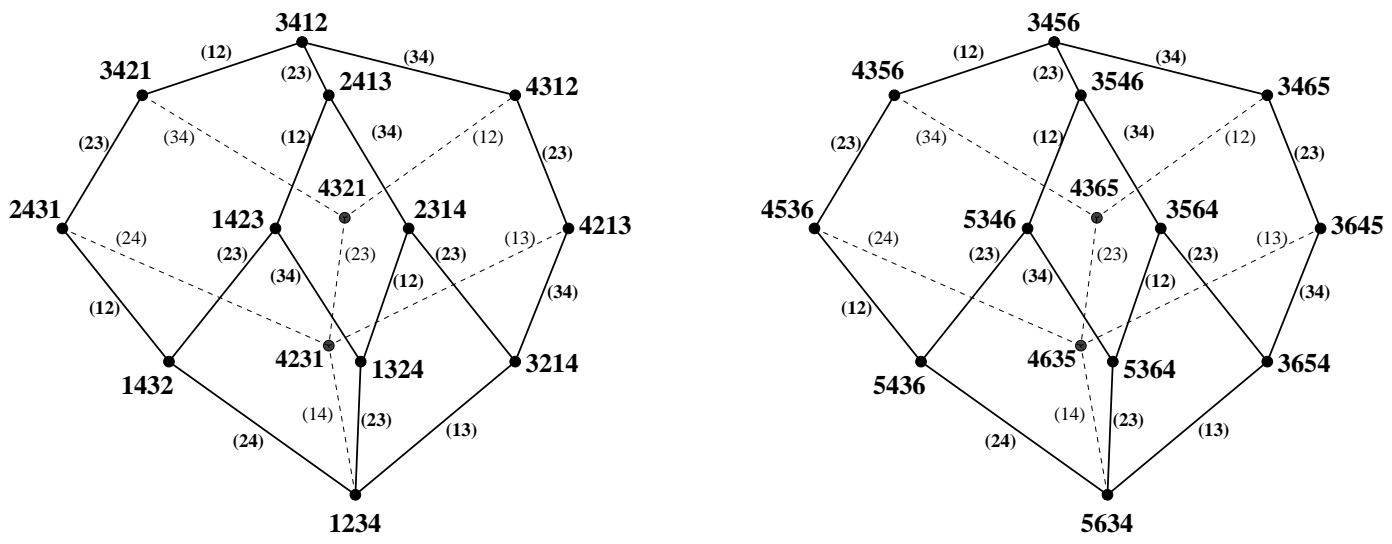

Figure 8. Two labelings of a bridge polytope. At the left, vertices are labeled by ordinary permutations, and edges are labeled by the pair of values which are swapped. At the right, vertices are labeled by affine permutations, and edges are labeled by the pair of positions which are swapped. The map from vertex-labels at the left to vertex-labels at the right is $z \mapsto \widetilde{z^{-1}}$. In both cases, the paths along the one-skeleton from top to bottom encode bridge decompositions of the permutation $\pi_{2,4}=(3,4,1,2)$.

Before giving the general construction, we present an example in Figure 8 , which encodes the bridge decompositions of plabic graphs with trip permutation $\pi=(3,4,1,2)$. The figure at the left shows the polytope obtained by taking the convex hull of the permutations

$$
\left.\left\{\left(z_{1}, z_{2}, z_{3}, z_{4}\right) \mid z_{1} \geq 1, z_{2} \geq 2, z_{3} \leq 3, z_{4} \leq 4\right)\right\} .
$$

The edge between two permutations is labeled by the values of the swapped entries. The figure at the right shows the same polytope, but now vertices are labeled by affine permutations. A vertex which was labeled by $z$ at the left is labeled at the right by the affinization $\widetilde{z^{-1}}$ of $z^{-1}$. Note that we took the inverse in order 
to get a vertex-labeling of the polytope such that edges correspond to the positions of the swapped entries, agreeing with the sequence of transpositions defined in Definition 2.17 .

The main result of this section will imply that the minimal chains in the one-skeleton of the bridge polytope from "top" to "bottom" (from $(3,4,1,2)$ to $(1,2,3,4)$ using the vertex-labeling at the left, and from $(3,4,5,6)$ to $(5,6,3,4)$ using the vertex-labeling at the right) are in bijection with the bridge decompositions of the reduced plabic graphs with trip permutation $\pi_{2,4}=(3,4,1,2)$. For example, the leftmost chain from top to bottom is labeled by the sequence of transpositions (12), (23), (12), (24); the corresponding plabic graph is shown in Figure 9.

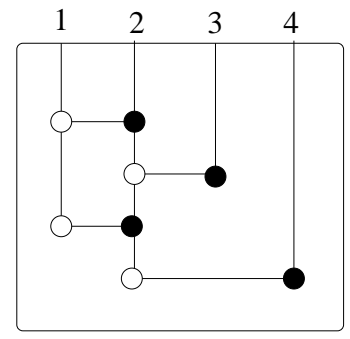

Figure 9. The plabic graph coming from the sequence of bridges (12), (23), (12), (24). Note that its trip permutation is $(3,4,1,2)=(24)(12)(23)(12)$.

We now turn to the general construction.

Definition 3.1. Let $J \subset\{1,2, \ldots, n\}$ and set

$$
S_{J}=\left\{\pi \in S_{n} \mid \pi(j) \geq j \text { for } j \in J \text { and } \pi(j) \leq j \text { for } j \notin J .\right\}
$$

In other words, any permutation in $S_{J}$ is required to have a weak excedance in position $j$ for each $j \in J$, and $a$ weak non-excedance in position $j$ for each $j \notin J$.

We define a polytope $\mathrm{Br}_{J}$ by

$$
\operatorname{Br}_{J}=\operatorname{Conv}\left\{(\pi(1), \pi(2), \ldots, \pi(n)) \mid \pi \in S_{J}\right\} \subset \mathbb{R}^{n} .
$$

In the special case that $J=\{1,2, \ldots, k\}$, we write

$$
\operatorname{Br}_{k, n}=\operatorname{Br}_{J}=\left\{\pi \in S_{n} \mid \pi(j) \geq j \text { for } 1 \leq j \leq k \text { and } \pi(j) \leq j \text { for } k+1 \leq j \leq n .\right\}
$$

Recall the definition of $\pi(J)$ from Definition 2.9 the decorated permutation labeling the corresponding TP Schubert cell. Let $e(J)$ be the decoration of the identity which has counterclockwise fixed points precisely in positions $J$.

The following is our main result. We describe it using the vertex-labeling of the bridge polytope with ordinary permutations, as in Definition 3.1, but it can be easily translated using the vertex-labeling by affine permutations.

Theorem 3.2. Let $J$ be a $k$-element subset of $[n]$. The shortest paths from $\pi(J)$ to the identity permutatione along the one-skeleton of the bridge polytope $\mathrm{Br}_{J}$ are in bijection with the $B C F W$-bridge decompositions of the permutation $\pi(J)^{-1}$, where a sequence of edge-labels in a path is interpreted as a sequence of transpositions $\tau$ in the bridge decomposition. Equivalently, there is an edge between two vertices $\pi$ and $\hat{\pi}$ of the bridge polytope $\mathrm{Br}_{J}$ if and only if there exists some pair $i<\ell$ such that $(i \ell) \pi=\hat{\pi}$, and $\pi(j)=\hat{\pi}(j)=j$ for $i<j<\ell$. In other words, $\pi$ and $\hat{\pi}$ differ by swapping the values $i$ and $\ell$, and $i+1, i+2, \ldots, \ell-1$ are fixed points of both $\pi$ and $\hat{\pi}$.

We will prove Theorem 3.2 in Section 4

The following corollary is immediate from Theorem 3.2

Corollary 3.3. The shortest paths in the bridge polytope $\mathrm{Br}_{k, n}$ from $\pi_{k, n}$ down to the identity e are in bijection with the BCFW-bridge decompositions of the permutation $\pi_{n-k, n}$. In other words, we can read off all BCFW-bridge decompositions of $\pi_{n-k, n}$ by recording the edge-labels on all shortest paths from $\pi_{k, n}$ to $e$ in the one-skeleton of $\mathrm{Br}_{k, n}$. 


\section{Bruhat interval polytopes and the proof of Theorem 3.2}

Bruhat interval polytopes are a class of polytopes which were recently studied by Kodama and the second author in KW13, in connection with the full Kostant-Toda lattice on the flag variety. The combinatorial properties of Bruhat interval polytopes were further investigated by Tsukerman and the second author in [TW14. We will show that bridge polytopes are isomorphic to certain Bruhat interval polytopes, and use a result from [KW13 as a tool for proving Theorem 3.2. We will also deduce a characterization of the one-skeleta of a large class of Bruhat interval polytopes.

Definition 4.1. Let $u, v \in S_{n}$ such that $u \leq v$ in (strong) Bruhat order. The Bruhat interval polytope $\mathrm{Q}_{u, v}$ is defined as the convex hull

$$
\mathrm{Q}_{u, v}=\operatorname{Conv}\left\{(z(1), \ldots, z(n)) \mid \text { for } z \in S_{n} \text { such that } u \leq z \leq v\right\} .
$$

Note that when $u$ is the identity and $v=w_{0}$, the longest permutation in $S_{n}$, the Bruhat interval polytope $\mathrm{Q}_{u, v}$ equals the permutohedron $\operatorname{Perm}_{n}$.

Lemma 4.2. The bridge polytope $\mathrm{Br}_{J}$ is isomorphic to the Bruhat interval polytope $\mathrm{Q}_{e, \pi(J)^{-1}}$. More specifically, recall that for $J$ a $k$-element subset of $[n]$, we write $J=\left\{j_{1}<\cdots<j_{k}\right\}$, and $J^{c}=\left\{h_{1}<\cdots<h_{n-k}\right\}$. Consider the map $\psi: \mathbb{R}^{n} \rightarrow \mathbb{R}^{n}$ defined by $\psi\left(x_{1}, \ldots, x_{n}\right)=\left(x_{h_{1}}, x_{h_{2}}, \ldots, x_{h_{n-k}}, x_{j_{1}}, x_{j_{2}}, \ldots, x_{j_{k}}\right)$. Then $\Psi$ is an isomorphism from $\mathrm{Br}_{J}$ to $\mathrm{Q}_{e, \pi(J)^{-1}}$.

Proof. Since $\psi$ simply permutes the coordinates of $\mathbb{R}^{n}$, it obviously acts as an isomorphism on any polytope. Moreover, $\psi$ takes the vertex $(z(1), \ldots, z(n))$ labeled by the permutation $z$ in $\operatorname{Br}_{k, n}$ to the vertex labeled by the permutation $z \pi(J)^{-1}$ of $\mathrm{Q}_{e, \pi(J)^{-1}}$. In particular, it takes the vertices $\pi(J)$ and $e$ of $\mathrm{Br}_{k, n}$ to the vertices $e$ and $\pi(J)^{-1}$ of $\mathrm{Q}_{e, \pi(J)^{-1}}$, respectively. It is not hard to show that $\psi$ maps the set of permutations $S_{J}$ to the set of permutations in the interval $\left[e, \pi(J)^{-1}\right]$.

Once we have established Theorem 3.2. Lemma 4.2 immediately implies the following description of the one-skeleton of any Bruhat interval polytopes $\mathrm{Q}_{e, w}$, when $w$ is a Grassmannian permutation.

Corollary 4.3. Let $w$ be a Grassmannian permutation in $S_{n}$, i.e. a permutation with at most one descent. Then there is an edge between vertices $u$ and $v$ in $Q_{e, w}$ if and only if $u$ and $v$ satisfy the following properties:

- $v=(i \ell) u$

- in the permutations $u$ and $v$, each of the values $i+1, i+2, \ldots, \ell-1$ are in precisely the same positions that they are in $w$.

We now prove Theorem 3.2 , by establishing Lemma 4.4, Proposition 4.6, and Theorem 4.7, below.

Lemma 4.4. If there is an edge in $\mathrm{Br}_{J}$ between $\pi$ and $\hat{\pi}$, then there exists some transposition $(i \ell)$ such that $(i \ell) \pi=\hat{\pi}$.

Proof. By [KW13, Theorem A.10], every edge of a Bruhat interval polytope connects two vertices $u$ and $v$, where $v=(i \ell)$ for some transposition $(i \ell)$. Lemma 4.4 now follows from Lemma 4.2,

Remark 4.5. It was shown more generally in [TW14] that every face of a Bruhat interval polytope is a Bruhat interval polytope.

Proposition 4.6. Suppose there's an edge in $\operatorname{Br}_{J}$ between $\pi$ and $\hat{\pi}$ where $(i \ell) \pi=\hat{\pi}$ for some $i<\ell$, i.e. $\pi$ and $\hat{\pi}$ differ by swapping the values $i$ and $\ell$. Then $i+1, i+2, \ldots, \ell-1$ must be fixed points of $\pi$ and $\hat{\pi}$.

Proof. We use a proof by contradiction. Suppose that not all of $i+1, i+2, \ldots, \ell-1$ are fixed points. Let $j$ be the smallest element of $\{i+1, i+2, \ldots, \ell-1\}$ which is not a fixed point. Let $a, b$, and $c$ be the positions of $i, \ell$, and $j$ in $\pi$.

Without loss of generality, $\pi_{c}=j>c$. So $c \in J$ and is a position of a weak excedance in any permutation in $S_{J}$. Since we have an edge in the polytope between $\pi$ and $\hat{\pi}$, there exists a $\lambda \in \mathbb{R}^{n}$ such that $\lambda \cdot x:=\sum_{h} \lambda_{h} x_{h}$ (applied to $x \in S_{J}$ ) is maximized precisely on $\pi$ and $\hat{\pi}$. In particular, we must have $\lambda_{a}=\lambda_{b}$.

If $\lambda_{a}=\lambda_{b}<\lambda_{c}$, then define $\tilde{\pi}$ so that it agrees with $\pi$ except in positions $b$ and $c$, where we have $\tilde{\pi}_{b}=j$ and $\tilde{\pi}_{c}=\ell$. Since $\pi_{b}=\ell$ and $\hat{\pi}_{b}=i$ and $i<j<\ell$, permutations in $S_{J}$ are allowed to have a $b$ th coordinate of $j$. Since $j>c$ and $j<\ell$, we have $\ell>c$. So permutations in $S_{J}$ are allowed to have a $c$ th coordinate of $\ell$. Therefore $\tilde{\pi} \in S_{J}$. But $\lambda \cdot \tilde{\pi}>\lambda \cdot \pi$, which is a contradiction. 
If $\lambda_{a}=\lambda_{b}>\lambda_{c}$, then define $\tilde{\pi}$ so that it agrees with $\pi$ except in positions $a$ and $c$, where we have $\tilde{\pi}_{a}=j$ and $\tilde{\pi}_{c}=i$. Since $\pi_{a}=i$ and $\hat{\pi}_{a}=\ell$ and $i<j<\ell$, permutations in $S_{J}$ are allowed to have an $a$ th coordinate of $j$. Since $\pi_{c}=j>c$, and $i+1, i+2, \ldots, j-1$ are fixed points of $\pi, c \notin\{i+1, i+2, \ldots, j-1\}$. So $c \leq i$, and permutations in $S_{J}$ are allowed to have a $c$ th coordinate of $i$ (recall that $c \in J$ and hence $c$ must be a weak excedance in any element of $S_{J}$ ). Therefore $\tilde{\pi} \in S_{J}$. But $\lambda \cdot \tilde{\pi}>\lambda \cdot \pi$, which is a contradiction.

Theorem 4.7. Consider the bridge polytope $\operatorname{Br}_{J}$ and two permutations $\pi$ and $\hat{\pi}$ in $S_{J} \subset S_{n}$ such that $(i \ell) \pi=\hat{\pi}$ and $i+1, i+2, \ldots, \ell-1$ are fixed points of $\pi$ and $\hat{\pi}$. Choose a vector $\lambda=\left(\lambda_{1}, \ldots, \lambda_{n}\right) \in \mathbb{R}^{n}$ whose coordinates are some permutation of the numbers $1, n^{2}, n^{4}, \ldots, n^{2(n-1)}$, and such that

$\lambda_{\pi^{-1}(1)}<\lambda_{\pi^{-1}(2)}<\cdots<\lambda_{\pi^{-1}(i-1)}<\square<\lambda_{\pi^{-1}(i)}=\lambda_{\pi^{-1}(\ell)}<\square<\lambda_{\pi^{-1}(\ell+1)}<\cdots<\lambda_{\pi^{-1}(n-1)}<\lambda_{\pi^{-1}(n)}$.

Here the $\square$ at the left represents the coordinates $\lambda_{\pi^{-1}(j)}$ for $j \in J$ and $i<j<\ell$, sorted from left to right in increasing order of $j$, and the $\square$ at the right represents the coordinates $\lambda_{\pi^{-1}(j)}$ for $j \notin J$ and $i<j<\ell$, sorted from left to right in increasing order of $j$.

Then when we calculate the dot product $\lambda \cdot x$ for each $x \in S_{J}, \lambda \cdot x$ is maximized precisely on $\pi$ and $\hat{\pi}$. In particular, there is an edge in $\mathrm{Br}_{J}$ between $\pi$ and $\hat{\pi}$.

Proof. To prove Theorem 4.7, we will first use the fact that the coordinates of $\lambda$ are $1, n^{2}, \ldots, n^{2(n-1)}$ to show that to solve for the $x \in S_{J}$ on which $\lambda \cdot x$ is maximized, it suffices to use the greedy algorithm. In other words, we will show that we can construct such $x$ by maximizing individual coordinates $x_{i}$ one by one, starting with the $x_{i}$ where $\lambda_{i}$ is maximal, and continuing in decreasing order of the values of $\lambda_{j}$. Next we will show that when we run the greedy algorithm, we will get precisely the outcomes $\pi$ and $\hat{\pi}$.

Let us show now that in order to maximize the dot product $\lambda \cdot x$ for $x \in S_{J}$, the greedy algorithm works. Recall that the coordinates of $\lambda$ are the values $1, n^{2}, \ldots, n^{2(n-1)}$ (in some order). Let $h \in[n]$ be such that $\lambda_{h}=n^{2(n-1)}$. We first claim that we need to maximize $x_{h}$ subject to the condition $x \in S_{J}$. Let $w_{h}$ be that maximum possible value. Let $y \in S_{J}$ be some other permutation with $y_{h} \leq w_{h}-1$. Then $\lambda_{h} x_{h}-\lambda_{h} y_{h} \geq n^{2(n-1)}$. And since the maximum difference of $x_{j}$ and $y_{j}$ is $n-1$, we have

$$
\sum_{1 \leq j \leq n, j \neq h} \lambda_{j} y_{j}-\sum_{1 \leq j \leq n, j \neq h} \lambda_{j} x_{j} \leq(n-1)\left(1+n^{2}+\cdots+n^{2(n-2)}\right) .
$$

Now note that since $n-1<n$ and $1+n^{2}+\cdots+n^{2(n-2)}$ has $n-1$ terms, we have $(n-1)\left(1+n^{2}+\cdots+n^{2(n-2)}\right)<$ $n \cdot n \cdot n^{2(n-2)}=n^{2(n-1)}$. It follows that $\lambda \cdot x>\lambda \cdot y$.

Next let $h^{\prime} \in[n]$ be such that $\lambda_{h^{\prime}}=n^{2(n-2)}$. Using the same argument, and the inequality $(n-1)(1+$ $\left.n^{2}+\cdots+n^{2(n-3)}\right)<n^{2(n-2)}$, it follows that to maximize $\lambda \cdot x$, we need to now choose the maximum value of $x_{h^{\prime}}$ subject to the condition $x \in S_{J}$. Continuing in this fashion, we see that this greedy algorithm will compute all $x \in S_{J}$ such that $\lambda \cdot x$ is maximized.

Now we will show that if we use the greedy algorithm, choosing coordinates $x_{j}$ for $x \in S_{J}$ in decreasing order of the corresponding value $\lambda_{j}$, we will get precisely the permutations $\pi$ and $\hat{\pi}$.

Step 1. Looking at the ordering of the coordinates of $\lambda$ as described in Theorem 4.7, we first need to maximize $x_{\pi^{-1}(n)}$, then $x_{\pi^{-1}(n-1)}, \ldots$, then $x_{\pi^{-1}(\ell+1)}$. Therefore we place $n$ in position $\pi^{-1}(n), n-1$ in position $\pi^{-1}(n-1), \ldots, \ell+1$ in position $\pi^{-1}(\ell+1)$. (Note that there exist permutations $x \in S_{J}$ with these coordinates in these positions - for example, $\pi$ and $\hat{\pi}$ are two examples of such permutations.)

Step 2. Next we need to maximize the values that we put in positions $\pi^{-1}(j)$, for $i<j<\ell$ and $\pi^{-1}(j)=j \notin J$. But these are positions of weak non-excedances in $S_{J}$, so the best we can do is to put fixed points there. Note that $\pi$ and $\hat{\pi}$ also have fixed points in these positions, so the $x$ we are building so far agrees with both $\pi$ and $\hat{\pi}$.

Step 3. Now we want to maximize the values that we can put in positions $\pi^{-1}(i)$ and $\pi^{-1}(\ell)$. The greatest unused value so far is $\ell$ so we can put that in either position $\pi^{-1}(\ell)$ (agreeing with $\pi$ ) or $\pi^{-1}(i$ ) (agreeing with $\hat{\pi}$ ).

Now let $j$ be the maximum value that we have not yet placed in any position of the $x$ we are building. If $i<j<\ell$, then necessarily $j \in J$, i.e. $j$ is a position where all permutations of $S_{J}$ must have a weak excedance. So we must make $j$ a fixed point in $x$ (the only other option is to place a value smaller than $j$ in position $j$ ). Similarly for any other $i<j<\ell$, we must set $x_{j}=j$.

Having done this, the maximum value that we have not yet placed in $x$ is $i$. So we now put $i$ in position $\pi^{-1}(i)$ (or position $\pi^{-1}(\ell)$ ). Note that the $x$ we are building agrees with either $\pi$ or $\hat{\pi}$ so far. 
Step 4. Finally we want to maximize the values that we place in positions $\pi^{-1}(i-1), \ldots, \pi^{-1}(1)$. The remaining unused values are $i-1, \ldots, 1$. So for $i-1 \geq j \geq 1$, we place $j$ in position $\pi^{-1}(j)$.

This greedy algorithm has constructed precisely two permutations, $\pi$ and $\hat{\pi}$, and clearly $\lambda \cdot \pi=\lambda \cdot \hat{\pi}$. Therefore if we consider the values $\lambda \cdot x$ for $x \in S_{J}, \lambda \cdot x$ is maximized precisely on $\pi$ and $\hat{\pi}$. It follows that there is an edge in $\operatorname{Br}_{J}$ between $\pi$ and $\hat{\pi}$.

\section{The TWO-DIMEnsional FACES OF BRIDGE POLYTOPES}

In this section we describe the two-dimensional faces of bridge polytopes, and explain how they are related to the moves for reduced plabic graphs.

Theorem 5.1. A two-dimensional face of a bridge polytope is either a square, a trapezoid, or a regular hexagon, with labels as in Figure 10.
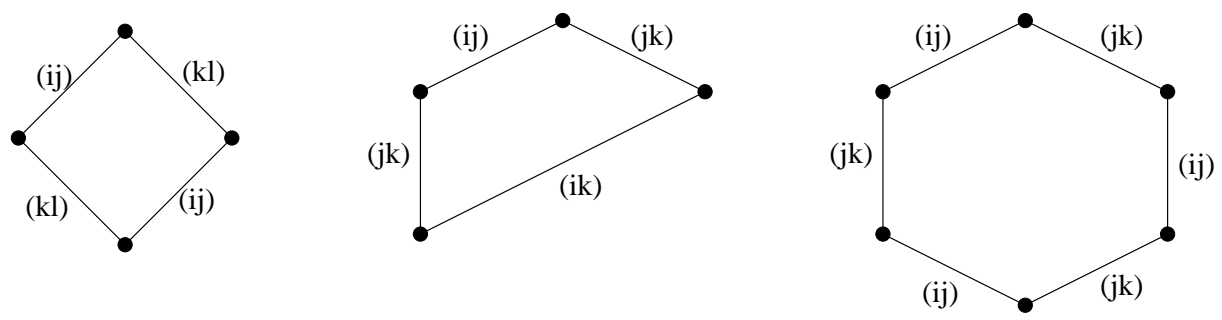

Figure 10. The possible two-dimensional faces of a bridge polytope. Here we have $i<$ $j<k<l$ for the square, $i<j<k$ or $k<j<i$ for the trapezoid, and $i<j<k$ for the hexagon.

Proof. Consider a two-dimensional face of a bridge polytope. By Lemma 4.4, each edge is parallel to a vector of the form $e_{i}-e_{\ell}$, and has length an integer multiple of $\sqrt{2}$. A simple calculation with dot products show that the possible angles among the edges are $\frac{\pi}{3}, \frac{\pi}{2}, \frac{2 \pi}{3}$ : more precisely, two vectors $e_{i}-e_{j}$ and $e_{k}-e_{l}$ (where $i, j, k, l$ are distinct) have angle $\frac{\pi}{2}$; two vectors $e_{i}-e_{j}$ and $e_{i}-e_{k}$ have angle $\frac{\pi}{3}$; and two vectors $e_{i}-e_{j}$ and $-e_{i}+e_{k}$ have angle $\frac{2 \pi}{3}$.

The only possibilities for such a polygon are: a square, a trapezoid (with angles $\frac{\pi}{3}, \frac{\pi}{3}, \frac{2 \pi}{3}, \frac{2 \pi}{3}$ ), a regular hexagon (all angles are $\frac{2 \pi}{3}$ ), an equilateral triangle, or a parallelogram. In the first three cases, the labels on the edges of the polygons must be as in Figure 10. In the case of the square, it follows from the rules of bridge decompositions that the intervals $[i, j]$ and $[k, l]$ must be disjoint, and hence without loss of generality $i<j<k<l$. (E.g. $k$ cannot lie in between $i$ and $j$, because in order to perform the swap $i$ and $j$, all elements between $i$ and $j$ must be fixed points. And we are not allowed to swap fixed points.) A similar argument explains the ordering on $i, j, k$ for the trapezoid and the hexagon.

We now argue that it is impossible for a two-dimensional face to be a triangle or a parallelogram. Note that if one traverses any cycle in the one-skeleton of a bridge polytope, the product of the corresponding edge labels must be 1. It follows that there cannot be a two-face with an odd number of sides, because the product of an odd number of transpositions is an odd permutation, and hence is never the identity. Therefore an equilateral triangle is impossible. Finally consider the case of a face which is a parallelogram. Its edge labels must have alternating labels $(i j),(i k),(i j),(i k)$. But the product $(i j)(i k)(i j)(i k)$ is not equal to the identity permutation.

Remark 5.2. The same proof shows that the face of any Bruhat interval polytope is a square, a trapezoid, or a hexagon, as shown in Figure 10.

Theorem 5.3. The three kinds of two-dimensional faces of bridge polytopes correspond to simple applications of the local moves for plabic graphs. More specifically, consider a shortest path $p$ from $\pi_{k, n}$ (or more generally $\pi(J))$ to e in the one-skeleton of the bridge polytope $\mathrm{Br}_{k, n}$ (or more generally $\mathrm{Br}_{J}$ ). Choose a two-dimensional face $F$ such that $p$ traverses half the sides of $F$, and modify $p$ along $F$, obtaining a new path $p^{\prime}$ which goes around the other sides of $F$. Then the reduced plabic graphs corresponding to $p$ and $p^{\prime}$ are related by homotopy and by the local moves for plabic graphs as in Figure 11. 
Proof. The proof of Theorem 5.3 is illustrated in Figure 11. The two reduced plabic graphs related by a square face in a bridge polytope are related by homotopy. The two plabic graphs related by a trapezoidal face are related by moves of type (M3). And the two plabic graphs related by a hexagonal face are related by a combination of moves (M1) and (M3). Note that in the latter case, the dashed edge in Figure 11 must be present, or else the plabic graph associated to our bridge decomposition would not be reduced.
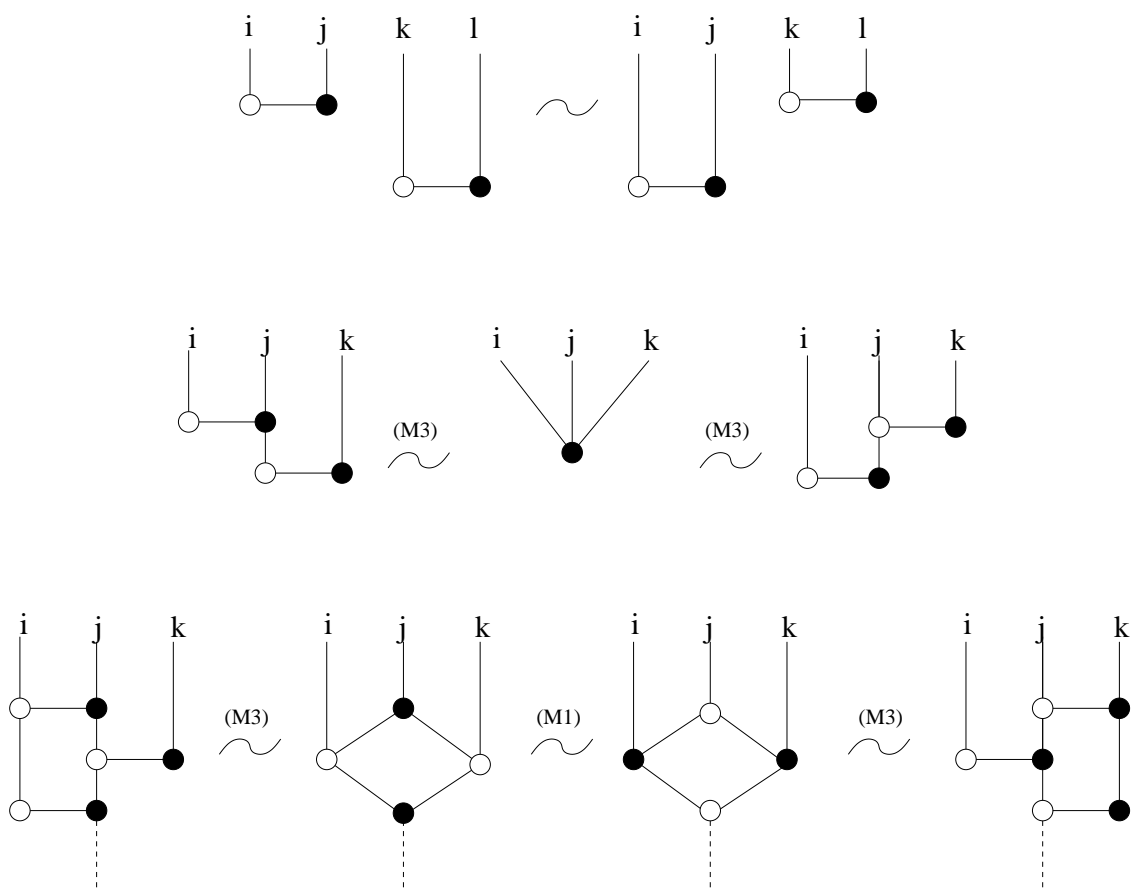

Figure 11. A graphical proof of Theorem 5.3 .

\section{REFERENCES}

$\left[\right.$ AHBC $\left.^{+} 12\right]$ Nima Arkani-Hamed, Jacob L. Bourjaily, Freddy Cachazo, Alexander B. Goncharov, Alexander Postnikov, and Jaroslav Trnka. Scattering amplitudes and the positive Grassmannian, 2012. Preprint, arXiv:1212.5605.

[BLVS+99] Anders Björner, Michel Las Vergnas, Bernd Sturmfels, Neil White, and Günter M. Ziegler. Oriented matroids, volume 46 of Encyclopedia of Mathematics and its Applications. Cambridge University Press, Cambridge, second edition, 1999.

[BW82] Anders Björner and Michelle Wachs. Bruhat order of Coxeter groups and shellability. Adv. in Math., 43(1):87-100, 1982.

[Ede81] Paul H. Edelman. The Bruhat order of the symmetric group is lexicographically shellable. Proc. Amer. Math. Soc., 82(3):355-358, 1981.

[KLS13] Allen Knutson, Thomas Lam, and David E. Speyer. Positroid varieties: juggling and geometry. Compos. Math., 149(10):1710-1752, 2013.

[KW11] Y. Kodama and L. K. Williams. KP solitons, total positivity, and cluster algebras. Proc. Natl. Acad. Sci. USA, 108(22):8984-8989, 2011.

[KW13] Y. Kodama and L. Williams. The full Kostant-Toda hierarchy on the positive flag variety, 2013. Preprint, arXiv: 1308.5011, to appear in Comm. Math. Phys.

[Lus94] G. Lusztig. Total positivity in reductive groups. In Lie theory and geometry, volume 123 of Progr. Math., pages 531-568. Birkhäuser Boston, Boston, MA, 1994.

[Pos06] Alexander Postnikov. Total positivity, grassmannians, and networks, 2006. Preprint, arXiv:math/0609764.

[Pro82] Robert A. Proctor. Classical Bruhat orders and lexicographic shellability. J. Algebra, 77(1):104-126, 1982.

[Rie99] Konstanze Rietsch. An algebraic cell decomposition of the nonnegative part of a flag variety. J. Algebra, 213(1):144154, 1999.

[Sco06] Joshua S. Scott. Grassmannians and cluster algebras. Proc. London Math. Soc. (3), 92(2):345-380, 2006.

[TW14] Emmanuel Tsukerman and Lauren Williams. Bruhat interval polytopes, 2014. Preprint, arXiv:1406.5202.

[Wil07] Lauren K. Williams. Shelling totally nonnegative flag varieties. J. Reine Angew. Math., 609:1-21, 2007.

Department of Mathematics, University of California, Berkeley, CA 94720-3840

E-mail address: williams@math.berkeley.edu 